\title{
Differences in systemic inflammation between cigarette and biomass smoke-induced COPD
}

\author{
This article was published in the following Dove Press journal: \\ International Journal of COPD \\ I September 2017 \\ Number of times this article has been viewed
}

\author{
Rafael Golpe' \\ Irene Martín-Robles' \\ Pilar Sanjuán-López' \\ Luis Pérez-de-Llano' \\ Carlos González-Juanatey ${ }^{2}$ \\ José L López-Campos ${ }^{3,4}$ \\ Elena Arellano-Orden ${ }^{4}$ \\ 'Respiratory Medicine Service, \\ ${ }^{2}$ Cardiology Service, University \\ Hospital Lucus Augusti, Lugo, \\ ${ }^{3}$ Medical-Surgical Unit of Respiratory \\ Diseases, University Hospital Virgen \\ del Rocío, Sevilla, ${ }^{4}$ Center for \\ Biomedical Research in Respiratory \\ Diseases Network, Carlos III Health \\ Institute, Madrid, Spain
}

Correspondence: Rafael Golpe Servicio de Neumología, Hospital Universitario Lucus Augusti, Unidad Administrativa 4-A, c/Dr Ulises Romero, I, 27002 Lugo, Spain

Tel +34982 296859

Email rafael.golpe.gomez@sergas.es
Background and objective: It is known that biomarkers of systemic inflammation are raised in COPD caused by tobacco (T-COPD) compared with healthy controls, but there is less information on the inflammatory status of subjects with COPD caused by biomass smoke (B-COPD). In addition, the possible (if any) differences in inflammation between both types of the disease are still not well known. The aim of this study was to assess the inflammatory profile in B-COPD and T-COPD.

Methods: A total of 20 subjects (15 men and five women) with T-COPD were matched one to one for sex, age and forced expiratory volume in $1 \mathrm{~s}\left(\mathrm{FEV}_{1}\right)$ to $20 \mathrm{~B}-\mathrm{COPD}$ patients. In all, 20 sex-matched healthy subjects with normal lung function without smoking history or biomass exposure were included as controls. The following biomarkers were measured: exhaled nitric oxide, serum IL-6, IL-8, IL-5, IL-13, periostin, surfactant protein-P, TNF- $\alpha$, $\mathrm{IgE}$, erythrocyte sedimentation rate, C-reactive protein and fibrinogen. Complete blood count was also obtained.

Results: The age of the subjects was $70.2 \pm 7.9$ years and $\mathrm{FEV}_{1} \%$ was $56.2 \% \pm 14.6 \%$. Most inflammatory biomarkers were higher in both types of COPD than in healthy controls. IL-6, IL-8 and IL-5 were significantly higher in T-COPD than in B-COPD, without other significant differences.

Conclusion: Both types of COPD are associated with high levels of systemic inflammation biomarkers. T-COPD patients have a higher systemic inflammatory status than the patients with B-COPD.

Keywords: biomass smoke, COPD, cytokines, inflammation, smoking

\section{Introduction}

COPD is a leading cause of mortality. Cigarette smoking is the main risk factor for COPD, but indoor air pollution related to biomass burning is also a significant risk factor for the disease. ${ }^{1}$ COPD caused by biomass smoke (B-COPD) has been described mainly in developing countries. However, biomass smoke exposure is also a concern in industrialized countries. ${ }^{2-4} \mathrm{~B}-\mathrm{COPD}$ has some differences compared to COPD caused by tobacco (T-COPD). Patients with B-COPD have a slower decline of forced expiratory volume in $1 \mathrm{~s}\left(\mathrm{FEV}_{1}\right)$ and a different distribution of phenotypes. ${ }^{5,6}$

Additionally, biomass and cigarette smoke have different inflammatory and proteolytic effects in the lung in animal models. ${ }^{7}$ A recent study compared women with T-COPD and B-COPD and suggested a different inflammatory profile for both types of the disease. ${ }^{8}$ B-COPD patients had higher levels of T-helper 2 (Th2) cells, while T-COPD subjects had higher quantities of T-helper 17 (Th17) cells. ${ }^{8}$ Another study suggested that asthma-COPD overlap (ACO) was more frequent in B-COPD than in T-COPD. ${ }^{9}$ This could be explained by a predominant Th2 inflammatory profile in 
B-COPD. However, this disparity seemed attributable to a different distribution of males and females within each COPD group. ${ }^{9}$

Although T-COPD primarily affects the lungs and airways, the disease is also characterized by low-grade systemic inflammation, and several inflammatory biomarkers have been associated with clinical outcomes in this entity. ${ }^{10}$ However, the possible systemic consequences of B-COPD are still not well known. In addition, the differences between T-COPD and B-COPD have not been fully understood so far. Therefore, a study that evaluates the effects of both types of COPD on biomarkers of systemic inflammation, in both men and women, is desirable.

The objective of the present study was to assess the inflammatory status and the possible differences in inflammatory biomarkers between men and women with T-COPD and B-COPD. The results of the present study will help us to understand the potential implications of both exposures in the clinical presentation of COPD.

\section{Methods}

\section{Setting}

The study was carried out at the COPD clinic of Hospital Universitario Lucus Augusti in Lugo, Spain. The hospital provides community health services to 220,000 people who live mainly in rural areas, where biomass fuels (wood smoke) have been traditionally used for cooking and home heating.

\section{Study design and subjects}

This is an observational, cross-sectional study. Participants were recruited from the patients who followed up at the COPD clinic of our hospital. Subjects were identified from a database maintained for clinical purposes. The inclusion criteria were age $\geq 40$ years and diagnosis of COPD in accordance with the Global Initiative for Chronic Obstructive Lung Disease (GOLD). ${ }^{11}$ The exclusion criteria were alpha-1 antitrypsin deficiency, active neoplasm, diagnosis of collagen vascular disease, pregnancy, renal failure, significant liver disease, human immunodeficiency virus infection, concomitant diagnosis of other significant respiratory diseases, clinical instability for 8 weeks before the study entry and exposure to inhaled drugs or other fumes different from biomass smoke or tobacco. Exposure to biomass or tobacco smoke was determined during a clinical interview. Subjects were classified into the B-COPD group if they had a history of at least 20 years of exposure to biomass, either used for cooking or as a home heating system, and were never-smokers. Subjects with a history of significant passive smoking were excluded.
Cumulative biomass smoke exposure was expressed as hours-year (the product of the number of years exposed to smoke and the average number of hours spent daily around the smoke source). Subjects were classified into the T-group if they had a smoking background, with a pack-year index $>10$, and they did not have a significant exposure to biomass smoke. A total of 20 T-COPD subjects were matched one to one for sex, age and post-bronchodilator (postBD) $\mathrm{FEV}_{1}$ to $20 \mathrm{~B}-\mathrm{COPD}$ patients. Age was matched via nearest neighbor matching with a maximum allowable difference of 5 years between two participants. FEV $_{1}$ was matched according to the ordinal (1-4) GOLD stage of severity of ventilatory obstruction. ${ }^{11}$ A total of 20 subjects, matched for sex, were included as healthy controls. The control population consisted of volunteers recruited among the hospital staff through advertisements, which did not reveal any a priori hypothesis of the study. They did not have a history of biomass smoke exposure; they had never smoked and had normal lung function.

\section{Lung function testing}

Subjects underwent pre-bronchodilator and postBD spirometry and plethysmographic lung volume and single-breath carbon monoxide uptake measurements, following the procedures recommended by the American Thoracic Society/European Respiratory Society task force using an integrated lung function testing equipment (MasterScreen Body, MasterScreen Pneumo, MasterScreen Difusión, Version JLAB 5.20.0.52; Viasys Healthcare GmbH, Höchberg, Germany). Reference values for spirometry were those recommended by the Spanish Society of Pulmonology and Thoracic Surgery for Spanish population. ${ }^{12}$ Exhaled nitric oxide (FeNO) was measured (NIOX MINO; Aerocrine AB, Solna, Sweden) at a mouth flow rate of $50 \mathrm{~mL} / \mathrm{s}$ for $10 \mathrm{~s}$. Oxygen saturation was measured using a finger pulse oximeter (Pulsox 3i; Minolta, Ramsey, NJ, USA).

\section{Laboratory data}

Venous blood was obtained in the morning after an overnight fast. The biomarkers IL-5, IL-13, periostin, IgE and eosinophil blood count were measured, which were chosen as markers of a Th2-type inflammation. TNF- $\alpha$, IL- 6 and IL- 8 were selected because they have been widely studied in COPD, and they might be related to poorer prognosis and to a higher risk of comorbidities. Surfactant protein-P (SP), produced in type II pneumocytes, was selected as a marker of lung injury. Other common inflammatory biomarkers such as erythrocyte sedimentation rate (ESR), C-reactive protein (CRP) and fibrinogen were also measured, because they 
are acute-phase reactants previously associated with COPD with prognostic implications. Alpha- 1 antitrypsin and complete blood count were determined as well. Blood samples $(5 \mathrm{~mL})$ were collected in $\mathrm{K} 3$-ethylenediaminetetraacetic acid (EDTA) tubes (Vacuette; Greiner Bio-One $\mathrm{GmbH}$, Kremsmünster, Austria). Samples were immediately centrifuged, and plasma aliquots were stored at $-80^{\circ} \mathrm{C}$ until immediately before analysis. Plasma IL-5, IL-6, IL-8, IL-13, TNF- $\alpha$, SP (R\&D Systems, Inc., Minneapolis, MN, USA) and periostin (CUSABIO, Wuhan, China) levels were measured using commercially available enzyme immunoassay kits according to the manufacturers' protocols.

\section{Statistical analysis}

Normal distribution of data was assessed using the D'Agostino-Pearson test. For continuous variables, comparisons between the two groups were made using the Student's $t$-test or Mann-Whitney $U$ test, as applicable. For comparisons between more than two groups, analysis of variance (ANOVA) or Kruskal-Wallis test was used. The chi-squared test was used for discrete variables. Data were reported as percentages for discrete variables and mean \pm standard deviation or median (interquartile range) for continuous variables, depending on whether they followed a normal distribution or not, respectively. Correlations between variables were assessed using Pearson's or Spearman's correlation coefficients, as appropriate. Significance tests were two tailed, and a $P$-value $<0.05$ was considered as statistically significant.

Sample size was estimated assuming that 1) the magnitude of the difference in the values of inflammatory biomarkers between B-COPD and control subjects would be similar to the difference in the CRP levels between T-COPD and control nonsmokers ${ }^{13}$ and 2) the difference in inflammation markers between B-COPD and T-COPD would be equivalent to the difference in Th2 cells between these types of disease ${ }^{14}$ (using these data with a two-sided 0.05 significance level and $95 \%$ power, a sample size of 20 subjects in each group would be large enough).

\section{Compliance with ethical standards}

The study was performed in accordance with the 1964 Declaration of Helsinki and its later amendments, and it was approved by our ethical committee (Comité Autonómico de Ética de la Investigación de Galicia, registry number: 2012/003). Written informed consent was obtained from all the subjects prior to the inclusion in the study.

\section{Results}

Figure 1 shows the flowchart of subject recruitment. A total of 15 men and five women in each group (B-COPD and T-COPD) were matched by postBD $\mathrm{FEV}_{1}$ and age. Two patients in the T-COPD group were current smokers, while the rest of the

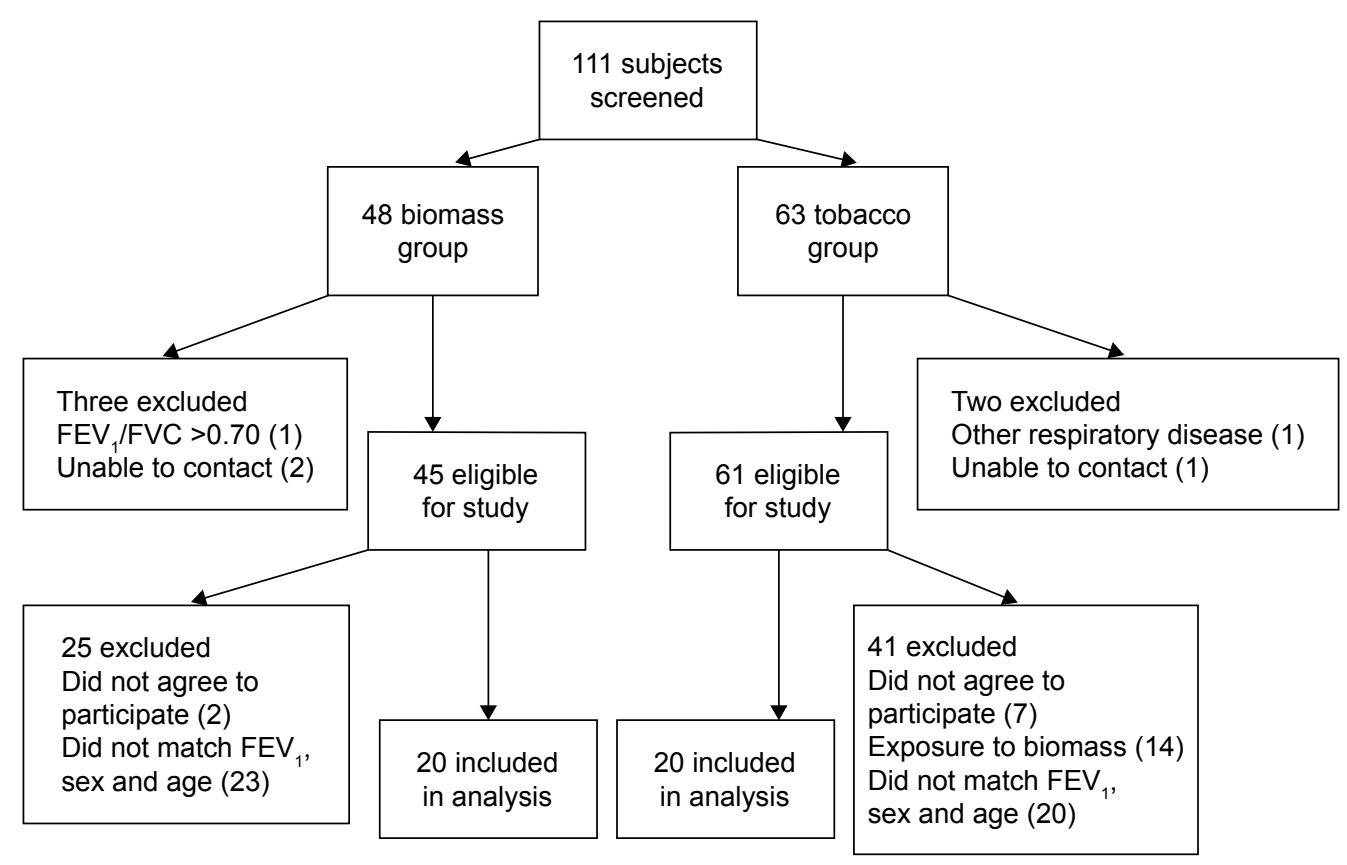

Figure I Flow diagram of subject recruitment.

Abbreviations: $\mathrm{FEV}_{1}$, forced expiratory volume in I s; FVC, forced vital capacity. 
Table I Clinical and demographic characteristics of the studied subjects

\begin{tabular}{|c|c|c|c|}
\hline Variable & $\begin{array}{l}\text { Biomass } \\
(n=20)\end{array}$ & $\begin{array}{l}\text { Tobacco } \\
(n=20)\end{array}$ & $P$-value \\
\hline Males & 15 & 15 & - \\
\hline Age, years & $70.2 \pm 8.6$ & $70.1 \pm 7.3$ & 0.72 \\
\hline Pack-years & - & $62.8 \pm 37.8$ & - \\
\hline Hours-year & $262.3 \pm 126.4$ & - & - \\
\hline $\mathrm{BMI}, \mathrm{kg} / \mathrm{m}^{2}$ & $31.5 \pm 5.1$ & $29.9 \pm 5.0$ & 0.31 \\
\hline Dyspnea, mMRC & & & 0.46 \\
\hline 0 & I (5) & I (5) & \\
\hline I & $7(35)$ & $10(50)$ & \\
\hline 2 & $10(50)$ & $9(45)$ & \\
\hline 3 & $2(10)$ & 0 & \\
\hline 4 & 0 & 0 & \\
\hline $\mathrm{SpO}_{2} \%$ & $96.2 \pm 1.8$ & $95.2 \pm 3.0$ & 0.24 \\
\hline FEV, preBD\% & $52.1 \pm 11.9$ & $49.9 \pm 17.1$ & 0.63 \\
\hline FVC preBD\% & $70.5 \pm 12.9$ & $75.4 \pm 16.4$ & 0.30 \\
\hline FEV,/FVC preBD\% & $54.7 \pm 9.8$ & $48.5 \pm 12.3$ & 0.08 \\
\hline FEV, postBD\% & $57.6 \pm 13.6$ & $54.4 \pm 16.4$ & 0.52 \\
\hline FVC postBD\% & $73.8 \pm 13.2$ & $79.5 \pm 15.3$ & 0.20 \\
\hline FEV / $/ F V C$ postBD\% & $57.7 \pm 11.1$ & $50.3 \pm 11.8$ & 0.06 \\
\hline Positive $\mathrm{BD}$ test & $8(40)$ & $6(30)$ & 0.96 \\
\hline DLCO\% & $84.1 \pm 21.7$ & $67.2 \pm 22.4$ & 0.02 \\
\hline DLCO/VA\% & $83.6 \pm 17.8$ & $62.6 \pm 18.5$ & 0.001 \\
\hline IC\% & $80.2 \pm 14.5$ & $70.1 \pm 16.0$ & 0.04 \\
\hline TLC\% & $103.1 \pm 10.6$ & $108.1 \pm 14.1$ & 0.22 \\
\hline RV\% & $|5| .5 \pm 3 \mid .7$ & $159.1 \pm 33.6$ & 0.47 \\
\hline $\mathrm{RV} / \mathrm{TLC} \%$ & $142.6 \pm 2 \mid .6$ & $145.5 \pm 19.6$ & 0.67 \\
\hline FeNO, ppb & $39.0 \pm 14.6$ & $27.6 \pm 16.3$ & 0.056 \\
\hline
\end{tabular}

Note: Results are expressed as mean \pm standard deviation or as number of observations (percentage).

Abbreviations: BMI, body mass index; mMRC, modified Medical Research Council; $\mathrm{SpO}_{2}$, pulse oxygen saturation; $\mathrm{FEV}_{1}$, forced expiratory volume in I s; preBD, pre-bronchodilator; FVC, forced vital capacity; postBD, post-bronchodilator; $\mathrm{BD}$, bronchodilator; DLCO, carbon monoxide diffusing capacity; DLCO/VA, carbon monoxide transfer coefficient; IC, inspiratory capacity; TLC, total lung capacity; $\mathrm{RV}$, residual volume; FeNO, exhaled nitric oxide. subjects in this group were ex-smokers. In all, 12 subjects in the B-COPD group were currently exposed to biomass, while eight patients had no current exposure to this smoke. Table 1 shows the clinical and demographic characteristics of the studied subjects. There were no significant differences between the two groups with respect to age, body mass index, dyspnea or oxygen saturation. Lung function test results were also similar for both groups, except inspiratory capacity (IC) and carbon monoxide diffusion variables, which were lower for the T-COPD group. There was a trend toward higher FeNO values in the B-COPD group, but the differences did not reach statistical significance.

Table 2 shows the laboratory data for the B-COPD and T-COPD groups and for the healthy controls. Almost all inflammatory biomarkers were significantly higher in both COPD groups than in controls. Differences between T-COPD and B-COPD were only significant in IL-6, IL-8 and IL-5, which were higher in the T-COPD group. Figure 2 compares the values of IL-6, IL-8 and IL-5 between groups. Biomarkers compatible with a Th2 inflammatory profile (IL-5, IL-13, eosinophil count, FeNO, periostin, $\operatorname{IgE}$ ) were not higher in B-COPD compared with T-COPD.

\section{Discussion}

This study has found that patients with B-COPD, similar to those with T-COPD, exhibit higher levels of inflammatory biomarkers than healthy subjects. In addition, the study has found differences in the systemic inflammatory profile of patients with B-COPD and T-COPD. Subjects with T-COPD exhibited

Table 2 Laboratory data

\begin{tabular}{|c|c|c|c|c|}
\hline Variable & Biomass $(n=20)$ & Tobacco $(n=20)$ & Controls $(n=20)$ & $P$-value ${ }^{a}$ \\
\hline IL-6 (pg/mL) & $1.68(1.08-2.00)^{b}$ & $3.84(2.04-7.67)^{\mathrm{b}}$ & $0.00(0.00-I .12)$ & $<0.001$ \\
\hline IL-8 (pg/mL) & $0.7 \mathrm{I}(0.0 \mathrm{I}-\mathrm{I} .68)^{\mathrm{b}}$ & $2.24(1.32-5.34)^{\mathrm{b}}$ & $0.22(0.00-2.04)$ & 0.005 \\
\hline IL-5 (pg/mL) & $1.45(1.15-1.83)^{c}$ & $2.08(1.38-2.48)^{c}$ & $1.60(0.99-2.20)$ & 0.03 \\
\hline IL-I3 (pg/mL) & $23.0(3.75-32.5)$ & $17.5(7.47-31.5)$ & $13.09(2.79-42.8)$ & 0.99 \\
\hline $\mathrm{SP}(\mathrm{ng} / \mathrm{mL})$ & $7.62(4.38-14.24)$ & $12.53(5.84-16.48)$ & $5.09(3.14-8.40)$ & 0.054 \\
\hline TNF- $\alpha(p g / m L)$ & $6.33(3.07-8.09)$ & $6.69(2.45-12.43)$ & $0.00(0.00-0.00)$ & $<0.001$ \\
\hline Periostin (ng/mL) & $0.42(0.26-0.66)$ & $0.42(0.33-0.80)$ & $0.00(0.00-0.00)$ & $<0.001$ \\
\hline Leukocyte count $(\mathrm{n}), 10^{3} / \mathrm{mL}$ & $7.81 \pm 1.72$ & $7.61 \pm 1.95$ & $6.54 \pm 2.13$ & 0.12 \\
\hline Neutrophil count $(\mathrm{n}), 10^{3} / \mathrm{mL}$ & $5.15(4.00-5.60)$ & $4.30(3.82-5.67)$ & $3.60(3.00-4.02)$ & $<0.001$ \\
\hline Neutrophils\% & $62.4 \pm 8.2$ & $61.7 \pm 6.7$ & $56.4 \pm 9.2$ & 0.054 \\
\hline Eosinophil count $(\mathrm{n}), \mathrm{mL}$ & $100.0(100.0-200.0)$ & $100.0(100.0-200.0)$ & $100.0(100.0-200.0)$ & 0.73 \\
\hline Eosinophils\% & $1.55(1.00-2.90)$ & $1.90(1.12-3.25)$ & $1.70(1.17-2.52)$ & 0.84 \\
\hline CRP & $2.80(1.50-5.86)$ & $3.17(2.44-8.83)$ & $0.58(0.13-1.01)$ & $<0.001$ \\
\hline ESR & $7.50(3.00-16.50)$ & $10.00(3.25-19.75)$ & $3.00(2.00-8.75)$ & 0.14 \\
\hline Fibrinogen & $396.0(343.0-45 I .0)$ & $387.0(326.0-445.0)$ & $328.0(313.0-368.0)$ & 0.01 \\
\hline Alpha-I antitrypsin & $152.5(131.5-172.5)$ & $132.5(\mid 16.0-149.0)$ & $126.0(109.0-133.0)$ & $<0.01$ \\
\hline $\lg \mathrm{E}$ & $52.7(20.3-237.5)$ & $68.2(15.0-212.5)$ & $16.1(6.9-62.8)$ & 0.09 \\
\hline
\end{tabular}

Notes: Results are expressed as mean \pm standard deviation or as median (interquartile range). ${ }^{\text {a }}$-values for results of ANOVA or Kruskal-Wallis tests for comparison between biomass, tobacco and control groups. ${ }^{b}$ Comparison between biomass and tobacco groups using Mann-Whitney $U$ test, $P<0.01$. ' ${ }^{C}$ Comparison between biomass and tobacco groups using Mann-Whitney $U$ test, $P=0.01$.

Abbreviations: CRP, C-reactive protein; ESR, erythrocyte sedimentation rate; ANOVA, analysis of variance; SP, Surfactant protein-P. 

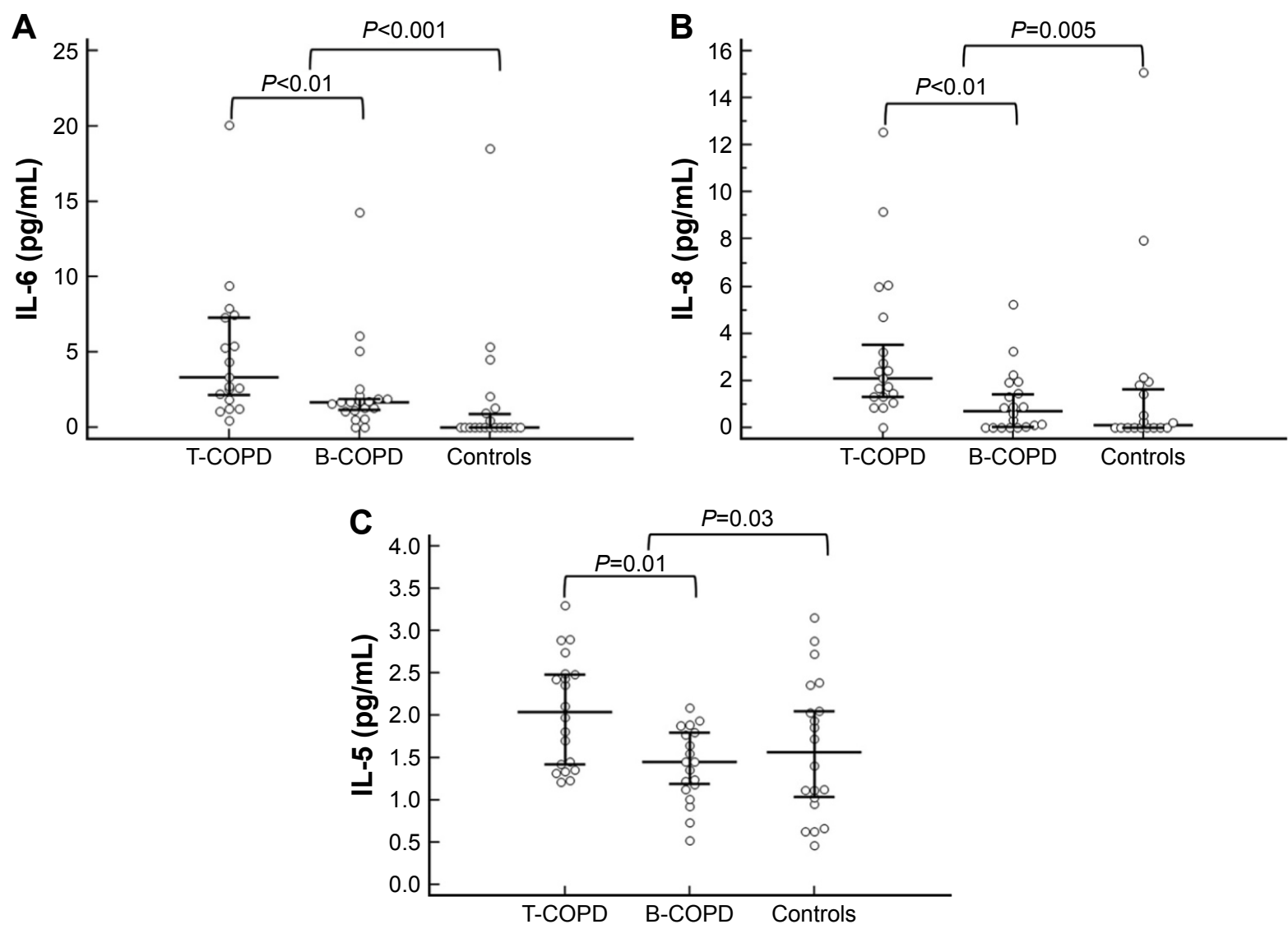

Figure 2 Differences between study groups in (A) IL-6, (B) IL-8 and (C) IL-5.

Note: The middle horizontal line represents the median, and the top and bottom horizontal lines represent $95 \%$ confidence interval for the median.

Abbreviations: T-COPD, COPD caused by tobacco; B-COPD, COPD caused by biomass smoke.

higher serum concentrations of several proinflammatory cytokines with potential prognostic significance. However, unlike a previous report, we did not find a predominant Th2type inflammation in B-COPD.

Persistent, low-level, systemic inflammation is thought to play a pathogenic role in COPD. ${ }^{15}$ The present study confirmed higher levels of most serum inflammatory biomarkers in COPD, caused by either biomass or tobacco smoke, compared with healthy controls. The information on the inflammatory status of B-COPD and the possible differences with T-COPD are presently limited. A previous paper found no differences in the level of CRP and matrix metalloproteinases between B-COPD and T-COPD. ${ }^{16}$ The present study has evaluated a substantial number of inflammation biomarkers, which shed light on the subject of inflammatory status in both types of the disease. The study is consistent with a previous report in which higher values of IL-6 and IL- 8 were found in T-COPD than in B-COPD. ${ }^{8}$ IL-6 has been associated with a higher risk of death in COPD, ${ }^{10}$ and it might have a role in the pathogenesis of atherosclerosis. ${ }^{17}$ Actually, IL-6 is raised in a cardiovascular comorbidity cluster of COPD patients. ${ }^{18}$
IL-8 might also have a role in cardiovascular disease. It could be implicated in the formation and preservation of the inflammatory environment of the damaged vascular wall. ${ }^{19}$ This raises the possibility of a higher risk for cardiovascular comorbidity in T-COPD compared with B-COPD. A recent preliminary study has found a higher prevalence of ischemic heart disease in patients with T-COPD, which would be congruent with this hypothesis. ${ }^{20}$

B-COPD and T-COPD exhibit clinical heterogeneity, and T-COPD subjects have a predominant emphysema phenotype. ${ }^{6}$ The present study is not an exception, and lower values of IC and carbon monoxide diffusion variables in T-COPD were found. In addition, ACO might be more frequent in B-COPD. ${ }^{9}$ Tobacco and biomass smoke might induce different inflammatory responses, which may account for the phenotypic differences between B-COPD and T-COPD. A role for T-helper $\left(\mathrm{CD}^{+}\right)$cells in the pathogenesis of obstructive lung diseases (COPD and asthma) has been suggested. Th1 lymphocytes have been implicated in the pathogenesis of smoking-related emphysema. ${ }^{21}$ On the other side, in asthma, there is a predominance of Th2 cells. ${ }^{22}$ Besides 
the classical Th1 and Th2 cells, continuing studies identified new subsets of $\mathrm{CD}^{+}$cells, such as Th17 cells, which are raised in the bronchial mucosa and peripheral blood of patients with COPD. ${ }^{23,24}$

A previous study by Solleiro-Villavicencio et $\mathrm{al}^{8}$ found that $\mathrm{CD} 4^{+} \mathrm{T}$-cell subpopulations of the Th2 type were more raised in the peripheral blood of subjects with B-COPD, while Th17 cells were higher in subjects with T-COPD. In contrast to these results, the present study has not found raised Th2-type biomarkers in peripheral blood of B-COPD subjects. No significant differences were found in FeNO values (a potential marker of a Th2-type airway inflammation) either. No clear significant correlations were found between Th2 biomarkers, which suggests that there were not subpopulations of subjects with a patent $\mathrm{Th} 2$ response.

In order to clarify the discrepancies between the present study and the findings of Solleiro-Villavicencio et al, we must draw attention to the fact that all the subjects in their study were females, while most of the patients in our study were males. A previous study showed that ACO was more frequent in B-COPD than in T-COPD, and this would be congruent with a Th2-predominant inflammation, but the differences seemed attributable to a different proportion of males and females in both types of the disease. ${ }^{9}$ Actually, ACO was more frequent in women, both in B-COPD and in T-COPD. ${ }^{9}$ Sex-related differences in the clinical expression of COPD have been reported, ${ }^{25,26}$ and it is plausible that women might exhibit a different inflammatory response to smoke, either produced by tobacco or biomass burning, than men. This possibility has not been sufficiently investigated. A recent study suggested that there are, indeed, sex differences in the inflammatory response to tobacco smoke. ${ }^{27}$ Whether these differences also exist concerning exposure to biomass smoke is presently speculative, although it seems highly plausible.

Another possible explanation for the discrepancies between the present study and the article by SolleiroVillavicencio et al might lie in differences in the kind of biomass fuels used. Animal models showed that mice subchronically exposed to cow dung burning exhibited predominantly increased neutrophil and macrophage chemokines. However, with wood smoke exposure, mice showed raised eosinophil cytokines. ${ }^{28}$ Consequently, the systemic inflammatory response might be different in distinct geographic areas depending on the kind of fuels used.

Finally, we must bear in mind that COPD is a very heterogeneous disease with various inflammatory phenotypes. For example, Khurana et $\mathrm{al}^{29}$ found that COPD patients with persistent sputum production have increased concentrations of some inflammatory mediators in the airways when compared to nonpersistent sputum producers. In addition, the present study included almost exclusively serum biomarkers, with the only exception of FeNO. These markers might not reflect adequately the inflammatory processes in the lung compartment. In T-COPD, there are few and weak correlations between lung and serum inflammatory biomarkers. ${ }^{30,31}$ Therefore, systemic inflammation does not seem to be a simple "overflow" of inflammatory components from the lung into the blood. ${ }^{30}$ Nevertheless, the present study offers insight into the subject of systemic inflammation in B-COPD and T-COPD, which is thought to be related to complications and comorbidities of the disease.

We did not find differences in other inflammatory biomarkers between B-COPD and T-COPD. SP is a pulmonaryspecific inflammatory marker that is raised in COPD patients compared with asthma patients. ${ }^{32}$ Because subjects in our study were matched by $\mathrm{FEV}_{1}$, it is understandable that the values of this biomarker of severity of lung injury were similar between groups. TNF- $\alpha, \mathrm{CRP}$ and fibrinogen were also similarly raised in B-COPD and T-COPD. We had expected to find higher values of TNF- $\alpha$ in the T-COPD group, because it seems to be primarily a marker of smoking (its values were higher in smokers without COPD than in COPD subjects in the ECLIPSE cohort), ${ }^{33}$ but it must be noted that only two subjects in the T-COPD group in the present study were current smokers, and this might account for the similar between-group values of this biomarker.

Several limitations of the present study must be acknowledged. We have only performed a single measurement of biomarkers, and it would be possible that the values of some biomarkers might vary over time. However, serum inflammatory markers seem to have acceptable repeatability in COPD. ${ }^{30}$ In addition, in order to avoid confounding variables, we matched subjects by age, sex and lung function. This requirement forced to exclude numerous patients from the study and, as a result, the participants might not be totally representative of the B-COPD and T-COPD patients seen in clinical practice. Biomass smoke exposure was self-reported and quantified according to the daily hours spent by the stove. This quantification might be somewhat imprecise, because it might fluctuate significantly according to seasonal variations within 1 year and also from year to year with changing climatic conditions. However, most epidemiologic and clinical studies on B-COPD did not use direct measurements of exposure, ${ }^{34}$ and the hours-year index is the most commonly used system to establish dose-response 
relationships. The values of the cytokines measured were low and of doubtful biological relevance. The study excluded subjects with significant comorbidities, so it is understandable that the values of the inflammatory biomarkers were lower than expected. Finally, socioeconomic status might differ for patients with B-COPD (who lived in rural areas) and T-COPD (who came mainly from urban settings). Consequently, lifestyle, diet or environmental variables might have biased the results to some degree.

\section{Conclusion}

Similar to patients with T-COPD, subjects with B-COPD exhibit a higher inflammatory status than healthy controls. There are some differences in the inflammatory profile of B-COPD and T-COPD. T-COPD patients have an increased systemic inflammatory status, including parameters potentially implicated in cardiovascular disease. However, we have not confirmed previous studies that suggested a Th2 polarization in B-COPD. Further studies are needed to define the possible sex differences in the biologic response to biomass and tobacco smoke and the potential disparities in the systemic consequences of both types of fumes.

\section{Acknowledgment}

This research was supported by a grant from Fundación Ramón Domínguez (Ref: O-023).

\section{Disclosure}

The authors report no conflicts of interest in this work.

\section{References}

1. Salvi S, Barnes PJ. Is exposure to biomass smoke the biggest risk factor for COPD globally? Chest. 2010;138:3-6.

2. Sood A, Petersen H, Blanchette CM, et al. Wood smoke exposure and gene promoter methylation are associated with increased risk for COPD in smokers. Am J Respir Crit Care Med. 2010;182(9):1098-1104.

3. Orozco-Levi M, García-Aymerich J, Villar J, Ramírez-Sarmiento A, Anto JM, Gea J. Wood smoke exposure and risk of chronic obstructive pulmonary disease. Eur Respir J. 2006;27(3):542-546.

4. López-Campos JL, Fernández-Villar A, Calero-Acuña C, et al; On-Sint Study Investigators. Occupational and biomass exposure in chronic obstructive pulmonary disease: results of a cross-sectional analysis of the On-Sint study. Arch Bronconeumol. 2017;53:7-12.

5. Ramírez-Venegas A, Sansores RH, Quintana-Carrillo RH, et al. FEV1 decline in patients with chronic obstructive pulmonary disease associated with biomass exposure. Am J Respir Crit Care Med. 2014;190(9): 996-1002.

6. Camp PG, Ramírez-Venegas A, Sansores RH, et al. COPD phenotypes in biomass smoke- versus tobacco smoke-exposed Mexican women. Eur Respir J. 2014;43(3):725-734.

7. Mehra D, Geraghty PM, Hardigan AA, Foronjy R. A comparison of the inflammatory and proteolytic effects of dung biomass and cigarette smoke exposure in the lung. PLoS One. 2012;7(12):e52889.
8. Solleiro-Villavicencio H, Quintana-Carrillo R, Falfán-Valencia R, Vargas-Rojas MI. Chronic obstructive pulmonary disease induced by exposure to biomass smoke is associated with a Th2 cytokine production profile. Clin Immunol. 2015;161(2):150-155.

9. Golpe R, Sanjuán-López P, Cano-Jiménez E, Castro-Añón O, Pérez-deLlano LA. Distribution of clinical phenotypes in patients with chronic obstructive pulmonary disease caused by biomass and tobacco smoke. Arch Bronconeumol. 2014;50:318-324.

10. Celli BR, Locantore N, Yates J, et al; ECLIPSE Investigators. Inflammatory biomarkers improve clinical prediction of mortality in chronic obstructive pulmonary disease. Am J Respir Crit Care Med.2012;185(10): 1065-1072.

11. Global Initiative for Chronic Obstructive Lung Disease (GOLD) [homepage on the Internet]. Global Strategy for the Diagnosis, Management and Prevention of COPD 2017. Available from: http://goldcopd. org/. Accessed June 12, 2017.

12. García-Río F, Calle M, Burgos F, et al. Normativa SEPAR. Espirometría. Arch Bronconeumol. 2013;49:388-401.

13. Pinto-Plata VM, Müllerova $\mathrm{H}$, Toso JF, et al. C-reactive protein in patients with COPD, control smokers and non-smokers. Thorax. 2006; 61(1):23-28.

14. Vargas-Rojas MI, Solleiro-Villavicencio H, Ramírez-Venegas A, Velázquez-Uncal M, Quintana R, Sansores-Martínez R. Differences in the polarization of the inflammatory response of patients with chronic obstructive pulmonary disease (COPD) secondary to smoking and to biomass smoke exposure. Eur Respir J. 2013;42(Suppl 57):595.

15. Fabbri LM, Rabe KF. From COPD to chronic systemic inflammatory syndrome? Lancet. 2007;370:797-799.

16. Montaño M, Sansores RH, Becerril C, et al. FEV1 inversely correlates with metalloproteinases 1, 7,9 and CRP in COPD by biomass smoke exposure. Respir Res. 2014;15:74.

17. Liu Y, Berthier-Schaad Y, Fallin MD, et al. IL-6 haplotypes, inflammation and risk for cardiovascular disease in a multiethnic dialysis cohort J Am Soc Nephrol. 2006;17:863-870.

18. Van Fleteren LE, Spruit MA, Groenen M, et al. Clusters of comorbidities based on validated objective measurements and systemic inflammation in patients with chronic obstructive pulmonary disease. Am J Respir Crit Care Med. 2013;187(7):728-735.

19. Apostolakis S, Vogiatzi K, Amanatidou V, Spanidos DA. Interleukin 8 and cardiovascular disease. Cardiovasc Res. 2009;84(3):353-360.

20. Golpe R, Martín-Robles I, Sanjuán-López P, et al. Prevalence of major comorbidities in chronic obstructive pulmonary disease caused by biomass smoke or tobacco. Respiration. 2017;94(1):38-44.

21. Grumelli S, Corry DB, Song LZ, et al. An immune basis for lung parenchymal destruction in chronic obstructive pulmonary disease and emphysema. PLoS Med. 2004;1(1):e8.

22. Holgate ST. Innate and adaptive immune responses in asthma. Nat Med. 2012;18(5):673-683.

23. Vargas-Rojas MI, Ramírez-Venegas A, Limón-Camacho L, Ochoa L, Hernández-Zenteno R, Sansores RH. Increase of Th17 cells in peripheral blood of patients with chronic obstructive pulmonary disease. Respir Med. 2011;105(11):1648-1654.

24. Di Stefano A, Caramori G, Gnemmi I, et al. T helper type 17-related cytokine expression is increased in the bronchial mucosa of stable chronic obstructive pulmonary disease patients. Clin Exp Immunol. 2009;157(2): 316-324.

25. De Torres JP, Casanova C, Hernández C, Abreu J, Aguirre-Jaime A, Celli BR. Gender and COPD in patients attending a pulmonary clinic. Chest. 2005;128(4):2012-2016.

26. Hong Y, Ji W, An S, Han SS, Lee SJ, Kim WJ. Sex differences of COPD phenotypes in nonsmoking patients. Int $J$ Chron Obstruct Pulmon Dis. 2016;11:1657-1662.

27. Faner R, González N, Cruz T, Graciela-Kalko S, Agustí A. Systemic inflammatory response to smoking in chronic obstructive pulmonary disease: evidence of a gender effect. PLoS One. 2014;9(5):e97491. 
28. Sussan TE, Ingole V, Kim JH, et al. Source of biomass cooking fuel determines pulmonary response to household air pollution. Am J Respir Cell Mol Biol. 2014;50(3):538-548.

29. Khurana S, Ravi A, Sutula J, et al. Clinical characteristics and airway inflammation profile of COPD persistent sputum producers. Respir Med. 2014;108(12):1761-1770.

30. Röpcke S, Holz O, Lauer G, et al. Repeatability of and relationship between potential COPD biomarkers in bronchoalveolar lavage, bronchial biopsies, serum and induced sputum. PLoS One. 2012;7(10):e46207.

31. Núñez B, Sauleda J, Garcia-Aymerich J, et al; Miembros del Grupo de Trabajo sobre la Caracterización Fenotípica y Evolución de la EPOC (PAC-COPD). Lack of correlation between pulmonary and systemic inflammation markers in patients with chronic obstructive pulmonary disease: a simultaneous, two-compartmental analysis. Arch Bronconeumol. 2016;52:361-367.
32. Akiki Z, Fakih D, Jounblat R, et al. Surfactant protein D, a clinical biomarker for chronic obstructive pulmonary disease with excellent discriminant values. Exp Ther Med. 2016;11(3):723-730.

33. Agustí A, Edwards LD, Rennard SI, et al; Evaluation of COPD longitudinally to Identify Predictive Surrogate Endpoints (ECLIPSE) Investigators. Persistent systemic inflammation is associated with poor clinical outcomes in COPD: a novel phenotype. PLoS One. 2012; 7(5):e37483.

34. Eisner MD, Anthonisen N, Coultas D, et al; on behalf of the Environmental and Occupational Health Assembly Committee on Nonsmoking COPD. An official American Thoracic Society public policy statement: novel risk factors and the global burden of chronic obstructive pulmonary disease. Am J Respir Crit Care Med. 2010;182(5): 693-718.

\section{Publish your work in this journal}

The International Journal of COPD is an international, peer-reviewed journal of therapeutics and pharmacology focusing on concise rapid reporting of clinical studies and reviews in COPD. Special focus is given to the pathophysiological processes underlying the disease, intervention programs, patient focused education, and self management protocols.

\section{Dovepress}

This journal is indexed on PubMed Central, MedLine and CAS. The manuscript management system is completely online and includes a very quick and fair peer-review system, which is all easy to use. Visit $\mathrm{http} / / / \mathrm{www}$.dovepress.com/testimonials.php to read real quotes from published authors. 